\title{
A strategy for improving water recovery in kimberlitic diamond mines
}

\author{
by A.J. Vietti*
}

\section{Synopsis}

Drought conditions and the possible lack of sufficient raw water supply are a constant reminder to the Southern African diamond miner of the need to minimize raw (new) water input while maintaining or expanding mine throughput.

Since water input volume is directly related to water output (loss) volume, mainly to the tailings, it is obviously beneficial to dewater the tailings as much as possible. However, this may not always be as easily achieved as first thought. Owing to the unique mineralogy of each kimberlite deposit and the unique chemical profile of each raw water source, dewatering of the slurry is not a standard process that can be applied equally across the entire diamond industry.

This paper presents a first-step strategy that kimberlite diamond mine operators should consider and apply to meet and exceed their watersaving targets.

\section{Keywords}

clays, colloids, thickening, water recovery, water conditioning.

\section{Introduction}

A common metric for measuring water use efficiency in a metallurgical plant is the amount of raw water used per ton of head feed treated $\left(\mathrm{m}^{3} / \mathrm{t}\right)$. The metric includes all water recycled from the dewatering process and from the tailings storage facility (TSF), and the only inputs are tons of ROM ore and cubic metres of raw water make-up to replace water lost to evaporation, seepage, and lock-up at the TSF (Figure 1).

The volume of new raw water available to the process is not unlimited and a maximum monthly allowance is typically set during the mine permitting process. Consequently, if the volume of water lost exceeds the volume of new water available or alternatively, in times of drought where the allowable volume is unavailable, the plant has no other option but to reduce throughput.

However, if the plant operator is able reduce the volume of water lost to the TSF, the plant will have additional capacity to either increase tonnage throughput or sustain nameplate capacity during periods of severe drought. The obvious conclusion, therefore, is to reduce the volume of water lost to the tailings by improving the efficiency of the dewatering process (typically gravity thickening) simply by increasing the density of the tailings being discharged. In practice, however, the unique mineralogy of each kimberlite deposit and the chemical profile of the raw water source used imply that at some mine sites, solid/liquid separation (or settling) of tailings slurries at the dewatering process is more easily achieved than at others. In these cases, simple practical steps such as firstly improving the flocculant reagent make-up and dosing control, and secondly focusing on thickener discharge control, are the only requirements for enhancing water recovery at the dewatering circuit. At other mine sites however, solid/liquid separation of the tailings may not be achieved at all, in which case efforts to increase water recovery by improving reagent dosage and thickener control would be fruitless unless the colloidal properties of the thickener feed slurry are modified.

This paper describes a strategy that can be implemented at most kimberlitic diamond mines to ensure that solid/liquid separation of the tailings slurry occurs efficiently and that the subsequent improvements in water recovery and quality (in terms of clarity) at the dewatering unit process can take place.

\section{Kimberlitic tailings solid/liquid separation: fundamentals}

Diamond processing, as with other mineral commodities, uses water in to assist in removing gangue minerals and concentrating the valuable products. Unlike most other ores, kimberlite contains a high proportion of clay minerals, which when wetted can interact with the plant process water to generate either settling or non-settling slurries. Notably, in

* Vietti Slurrytec (Pty) Ltd, South Africa.

(C) The Southern African Institute of Mining and Metallurgy, 2019. ISSN 2225-6253. This paper was first presented at the Diamonds - Source to Use 2018 Conference, 11-13 June 2018, Birchwood Hotel and OR Tambo Conference Centre, JetPark, Johannesburg, South Africa. 


\section{A strategy for improving water recovery in kimberlitic diamond mines}

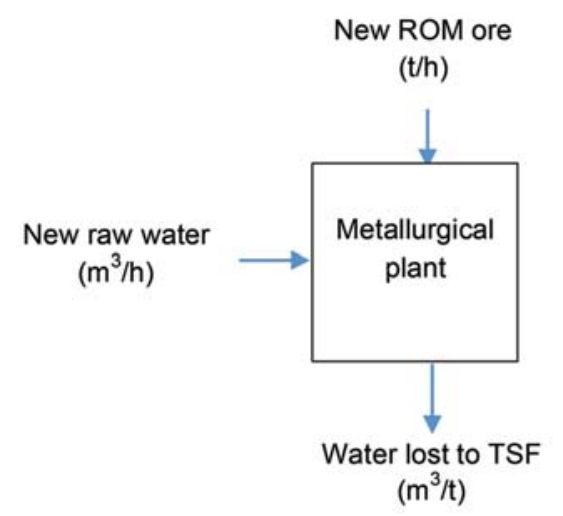

Figure 1-Raw water input and output model

most cases the dominant clays belong to the 2:1 smectite or swelling group of clays and the settling or solid/liquid separation properties of these slurries are determined largely by the chemical quality of the process water. Consequently, controlling the chemical conditions in the process water circuit is the first step in improving plant water recovery.

Before describing a strategy for controlling the chemical quality of the process water it is necessary to describe the fundamental mechanisms by which kimberlitic clay slurries are generated and which control their colloidal behaviour.

\section{Controlled and uncontrolled slurry dispersion}

In a typical kimberlite metallurgical circuit, the first contact between freshly mined ore and the plant process water is at the scrubbing or milling unit process. This step is critical in determining the colloidal characteristics of the resulting slurry, since the clays within the ore absorb water and other polar molecules between their unit layers, which initiates the swelling and ultimately the dispersion process. Two forms of swelling are known, which depend on the moisture content to which the clays are exposed.

Firstly, under conditions of low moisture content a limited stepwise expansion of the clay unit layers, known as interlayer (or type I) swelling, begins. This form of swelling occurs once the virgin ore is exposed to atmospheric moisture after blasting or crushing. Water molecules are drawn between the dehydrated clay platelets and up to three layers of water molecules are covalently bonded to the tetrahedral surfaces in a semi-crystalline structure that resembles that of ice. This mode of swelling leads to at most a doubling in the volume of the dry clay and may be responsible for a weakening of the rock matrix (Grimm, 1968).

The second form of swelling occurs at saturation (i.e. during the scrubbing or milling process) and can lead to an unlimited or complete separation of individual clay layers. This is known as osmotic (or type II) swelling. Under this condition, the exchangeable cations dissociate from the clay surface and move to the hydrated region between the clay particles; as such, they are regarded as being 'in solution' and hence lower the activity of the water between the particles. This allows more water from the surroundings to move into the interlayer region by osmotic forces, thereby increasing the interlayer swelling. This form of swelling may continue indefinitely and result in a slurry suspension in which normal electrical double layers separate the individual clay particles from one another (Figure 2).
The degree of osmotic swelling depends largely on the nature and concentration of the cations in the contacting water and the degree of octahedral substitution of the clay type. Monovalent cations in low concentration tend to cause unlimited swelling, a condition commonly referred to as uncontrolled dispersion, since they are small and can 'dissolve' more easily in the semi-crystalline water layers that surround the clay particles, thereby drawing more water between the adjacent particles by osmotic action. Divalent cations, on the other hand, tend to cause limited swelling since they have a disruptive effect on the structure of the water layer and can provide links between charged sites on adjacent silicate sheets. This condition is commonly referred to as controlled dispersion (Sequet, de la Calle, and Pezerat, 1975; Mering, 1946).

From a practical point of view, kimberlite mines that draw their raw water from surface sources such as rivers, dams, or lakes will tend to introduce good quality water into the process water circuit, which will result in the process water having a relatively low cation content and low conductivity. This quality of process water will promote uncontrolled dispersive conditions and the resulting slurries will tend to respond poorly, or not at all, to anionic polymer flocculant dosing, and even after excessively high flocculant doses the recovered thickener overflow clarity is generally poor (Figure 3 ). Under this condition it is normally necessary to dose a cationic polymer coagulant before flocculant addition to achieve solid/liquid separation and good overflow clarity. As a result, operating costs are high.

On the other hand, kimberlite mines that draw their raw water from deep wellfields or brackish water sources will have process water circuits with elevated cation contents and high conductivities. This quality of process water inhibits clay swelling and dispersion, resulting in slurries that are easily flocculated at low doses without the need for additional coagulant dosing. Recovered thickener overflow will have excellent clarity, and there are further beneficial spin-offs such as better thickener underflow density and lower underflow yield strengths, due to the absence of excessive amounts of polymer reagent (Figure 3).

\section{Slurry colloidal mechanisms}

Soil scientists and agriculturalists have long recognized the effects of water quality on the behaviour of the clay component in a soil (Mitchell and Soga, 2005; Richards,

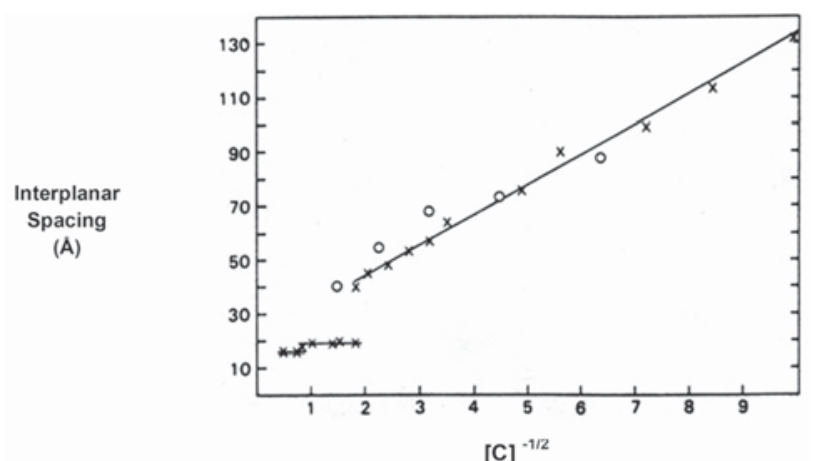

Figure 2-Change in layer spacing of a 2:1 clay with increasing $\mathrm{NaCl}$ concentration - type I and type II swelling (Grimm, 1968) 


\section{A strategy for improving water recovery in kimberlitic diamond mines}

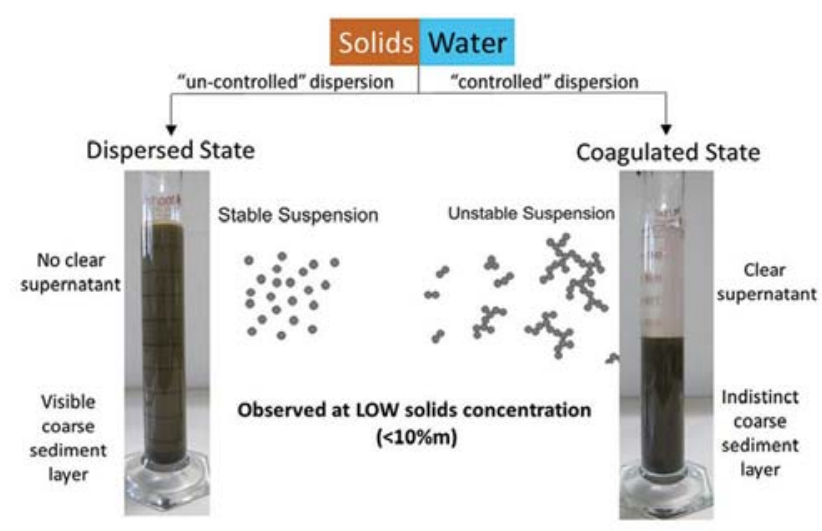

Figure 3-Slurry colloidal behaviour after controlled and uncontrolled dispersion

1969). To understand the mechanisms controlling these clay/water interactions, a diagnostic system using three criteria was developed, which enables the soil scientist to determine the potential deleterious effect of the water on the soil (Richards, 1969). These criteria can equally be applied to kimberlitic ores to indicate the slurry dispersive potential which the raw/process waters may impart to a particular ore type.

\section{Cation ratios}

The cation exchange capacity (CEC) is the total capacity of a soil (or kimberlite ore) to hold exchangeable cations (irrespective of type or valence), in meq/100 $\mathrm{g}$ and is directly related to the specific surface charge and charge density of the clays in the ore (Mitchell and Soga 2005). Once the CEC has been determined, the proportion of sodium ions which are adsorbed onto the surfaces of clays is a useful parameter to know. This has an important influence on the colloidal behaviour of the slurry and can be expressed as the exchangeable sodium percentage (ESP) of the suspension, defined as:

$$
E S P=\frac{N a^{+}}{C E C} \times 100
$$

This measure provides an indication of slurry sodicity. Slurries exhibiting high ESP values tend to be alkaline and colloidally dispersive, while slurries with low ESP values tend to be naturally coagulated and settling (Figure 4).
However, in practice, determining the ESP of the ore is not a simple procedure and is prone to experimental error due to mineral contaminants in the ore. A simpler method is to measure the cation content of the contacting water. The sodium adsorption ratio (SAR) value of the contacting water can then be used instead of the ESP to determine whether a process water is likely to generate slurries which are dispersive or not. The SAR is defined as:

$$
S A R=\frac{N a^{+}}{\sqrt{\left(\mathrm{Ca}^{2+}+M g^{2+}\right) / 2}}
$$

Since the SAR is easily derived from a normal chemical analysis of the process water (in meq/l) and since it is related to the ESP, it is more widely used. Process waters with SAR values greater than approximately 10 are likely to generate uncontrolled dispersive slurries, while those less than 10 are likely to generate coagulated or settling slurries (Mitchell and Soga, 2005; Richards, 1969).

\section{Slurry $\mathbf{p H}$}

Once in an uncontrolled dispersive state, the $\mathrm{pH}$ of the slurry is the second factor which can control coagulation and solids/liquid separation. Since clay particles have a plate-like morphology, the charges at the particle face and edge may be of similar or opposite sign, depending on the slurry $\mathrm{pH}$. These changes in charge and charge distribution at the clay surface can also affect the slurry solid/liquid separation behaviour.

The dominant surface of a clay particle is the flat face, which has a permanent negative charge due to imperfections in the clay crystal lattice (isomorphous substitution), which results in excess negative charge at the face. This excess negative charge is compensated by the adsorption of cations onto the crystal lattice surface (Figure 5). Once in a slurry state, these compensating cations may be exchanged with cations from the solution, depending on how strongly they are bound to the crystal surface. For this reason, they are known as exchangeable cations and their concentrations can be used as a measure of the amount of lattice charge, or cation exchange capacity (CEC), of the clay (van Olphen, 1977).

Clay particle edges, on the other hand, can be positively or negatively charged depending on the $\mathrm{pH}$ of the slurry. Under acidic conditions, hydroxyl- $\left(\mathrm{OH}^{-}\right)$-bearing groups at the clay crystal edge become protonated to carry an overall positive charge, leading to edge-to-face attraction which may
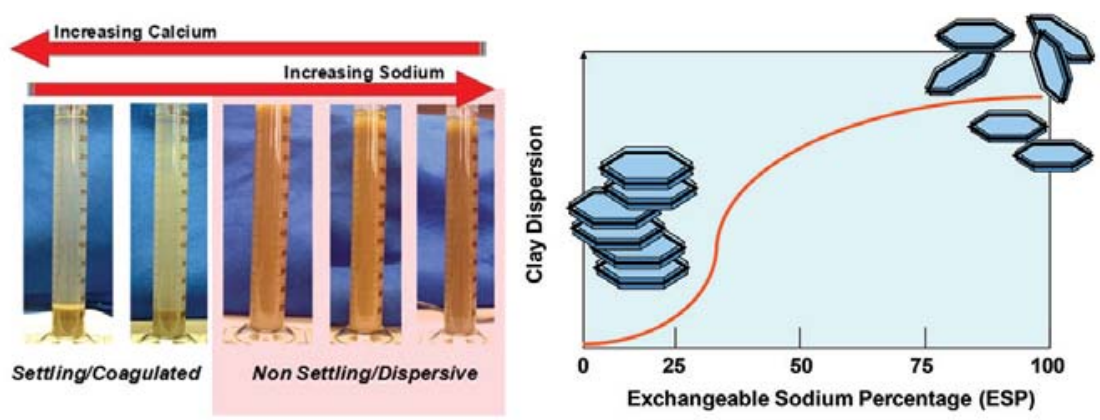

Figure 4-Effect of increasing sodicity on the suspension behaviour of a kimberlitic clay suspension 


\section{A strategy for improving water recovery in kimberlitic diamond mines}
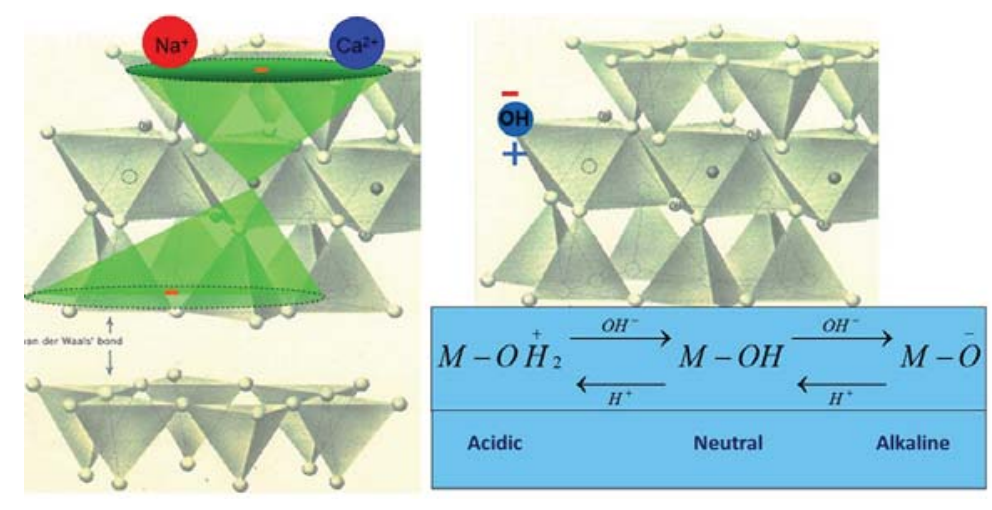

Figure 5-Typical 2:1 clay surface and edge charge
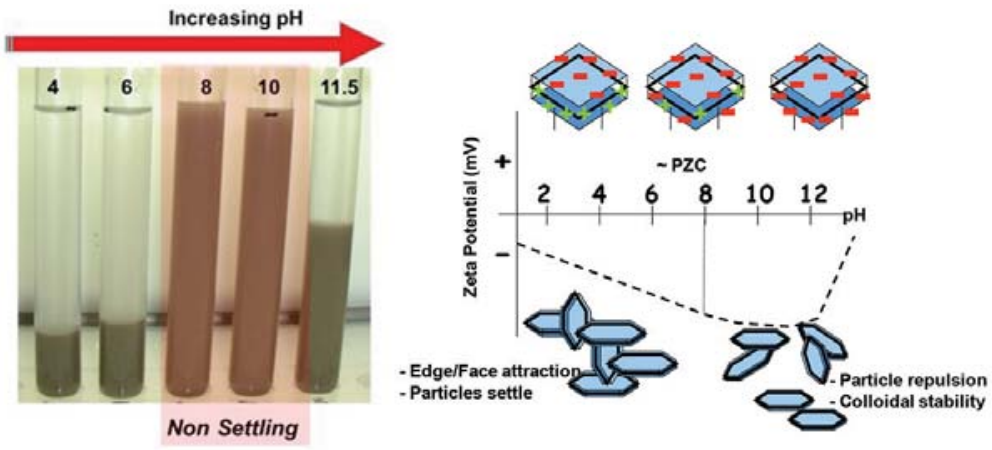

Figure 6-Effect of $\mathrm{pH}$ on the dispersive behaviour of a kimberlitic clay suspension

result in particle aggregation. Under alkaline conditions, the $\mathrm{OH}$ - groups will become deprotonated until an overall negative edge charge dominates, leading to edge-to-face repulsion and causing the clay particles to become colloidally dispersed (Svarovsky, 1981) (Figure 5).

The $\mathrm{pH}$ of a kimberlite clay suspension therefore greatly affects the charge associated with the clay particle edges. At low $\mathrm{pH}$, tailings slurries are coagulated naturally and settle under gravity, while at high suspension $\mathrm{pH}$ they tend to remain dispersed and non-settling (Figure 6).

\section{Slurry ionic strength}

The third and overriding mechanism for controlling the colloidal properties of a slurry is the absolute ionic concentration. This is commonly defined by the conductivity of the slurry and is typically measured in units of $\mathrm{mS} / \mathrm{cm}$.

The interaction between adjacent particles in dispersed colloidal systems is described by the Derjaguin, Landau, Verwey, and Overbeek (DLVO) theory of colloid stability and is based on the interaction between the van der Waals attractive force and the diffuse double layer repulsion force occurring between approaching particles (Figure 7). At low conductivity, a net potential energy barrier presents an obstacle to aggregation and the particles remain in a dispersed state. At moderate conductivity levels, the height of the energy barrier is reduced, and the particles may remain weakly coagulated in a secondary minimum well. As the ionic content of the dispersion is increased a point is reached where the energy barrier disappears, and the particles are spontaneously coagulated (Figure 8). This ionic concentration is known as the critical coagulation concentration (CCC) (Gregory, 1989).

The CCC can equally be termed the critical coagulation conductivity (in $\mathrm{mS} / \mathrm{cm}$ ), which would be the process water conductivity at which coagulation occurs for a particular ore type and at which solid/liquid separation is achieved. This is a measurable variable which can be used in a feedback loop to control the conductivity at which a process water circuit should be maintained, and forms the basis of the strategy for improving water recovery.

\section{Process water circuit conditioning strategy}

An example of the effectiveness of a process water conditioning strategy for improving water recovery and quality is provided below by way of case studies for two kimberlite mines in Southern Africa.

Mine A derives its raw water supply from shallow boreholes located along a major river course. Consequently, the plant process water circuit has a relatively low average conductivity. The mine suffers from periods during the year in which the process water clarity deteriorates and additional polymer coagulant dosing is required ahead of flocculation to clarify the thickener overflow.

The process water clarity issues are not related only to the ore types being treated; they are also a seasonal phenomenon. During the summer rainfall months, an increase in the use of polymer coagulant reagent use has been recorded, while during the winter season, the operation of the thickener tends to improve, resulting in better overflow clarity and lower reagent costs (Figure 9). 


\section{A strategy for improving water recovery in kimberlitic diamond mines}

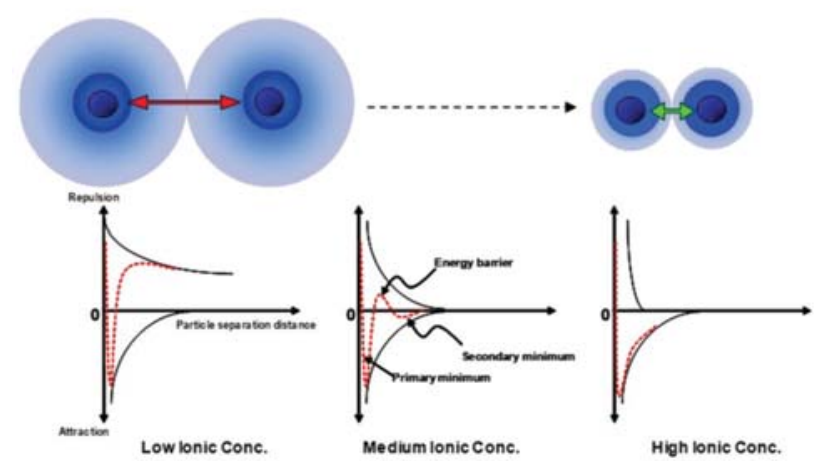

Figure 7-Net potential energy curve between two particles in suspension at three electrolyte concentrations

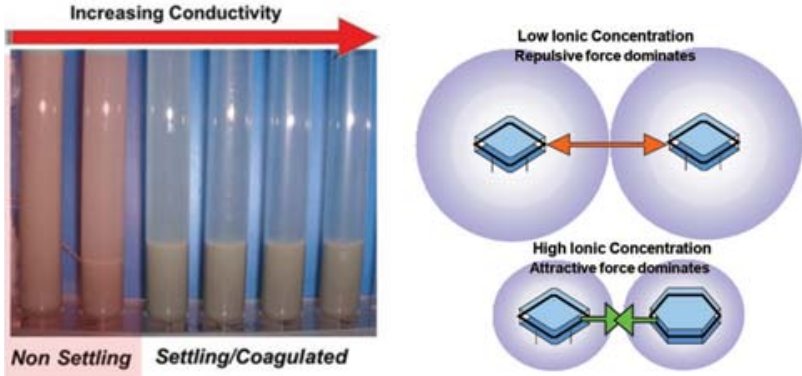

Figure 8-Effect of increasing salinity on the suspension behaviour of a kimberlitic clay suspension

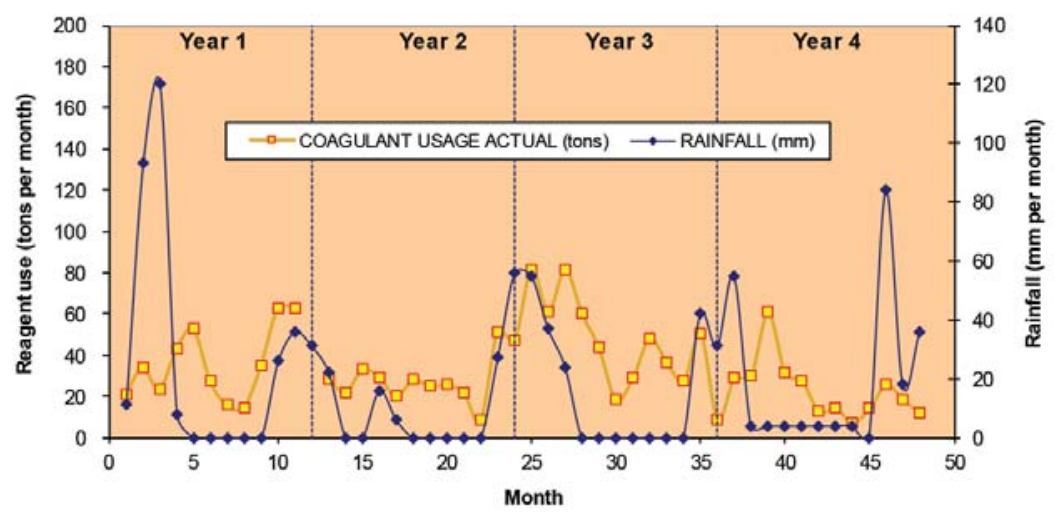

Figure 9-Correlation between coagulant consumption and seasonal rainfall at a kimberlite mine

These observations appear to be directly related to the seasonal wetting and drying cycle to which the kimberlitic ore in the pit or in stockpiles is exposed. During the summer season, the clays undergo uncontrolled dispersion when wetted with zero-conductivity rainwater in situ. When introduced to the plant, the process water conductivity is insufficient to overcome the CCC of the clays and often additional coagulant dosing is required before flocculation becomes effective.

During the winter season, the ore reporting to the plant is either dry or has not been previously wetted. When this ore is contacted with the plant process water, the clays therein undergo controlled dispersion since the conductivity of the water exceeds the CCC of the clays. Additional coagulant dosing ahead of flocculation is therefore not required (Figure 10).

To accommodate the seasonal change in clay dispersion properties and the varying CCC requirement of clays in different ore types, a minimum conductivity threshold for the mine process water circuit needs to be set, at which controlled dispersive slurries will be generated when any ore type is treated.

Vietti Slurrytec (VST) has developed the ClariVie44® process water conditioner, which is a blend of specific cations designed to increase the conductivity of process water without introducing environmental or corrosive pollutants. Since the process water conditioner is not polymer-based, it is recycled in the process water circuit without the need for continuous dosing. ClariVie44 ${ }^{\circledR}$ is dosed into the process water circuit to increase the conductivity; thereafter, smaller top-up doses are required to compensate for the diluting effect of the raw water make-up (Figure 11).

To test the principle, a notoriously difficult ore from kimberlite mine A was contacted with plant process water that had been treated with ClariVie $44{ }^{\circledR}$ to increasing the conductivity. The dispersion and natural settling behaviours were measured as illustrated in Figure 12.

At process water conductivities below $3 \mathrm{mS} / \mathrm{cm}$ (plant process water conductivity was measured at $2.9 \mathrm{mS} / \mathrm{cm}$ ), uncontrolled dispersion was encouraged, resulting in the establishment of colloidally stable suspensions. At higher conductivity, controlled dispersion was favoured, resulting in the formation of colloidally unstable suspensions which achieved better clarity.

In the case of mine A, the recommended strategy would be to maintain the process water circuit conductivity at a minimum of $3.3 \mathrm{mS} / \mathrm{cm}$ to ensure that effective solid/liquid separation after flocculation occurs at the thickener.

A similar strategy has been employed at another kimberlite diamond mine (mine B) since January 2017. In this case, raw water to the plant is sourced only from rainfall or snowmelt. The pristine quality of the raw water is, unfortunately, detrimental to the recovery of clear overflow from the dewatering thickener. ClariVie44 ${ }^{\circledR}$ has been implemented to condition the process water circuit to a minimum conductivity target of $1.2 \mathrm{mS} / \mathrm{cm}$, with excellent 


\section{A strategy for improving water recovery in kimberlitic diamond mines}

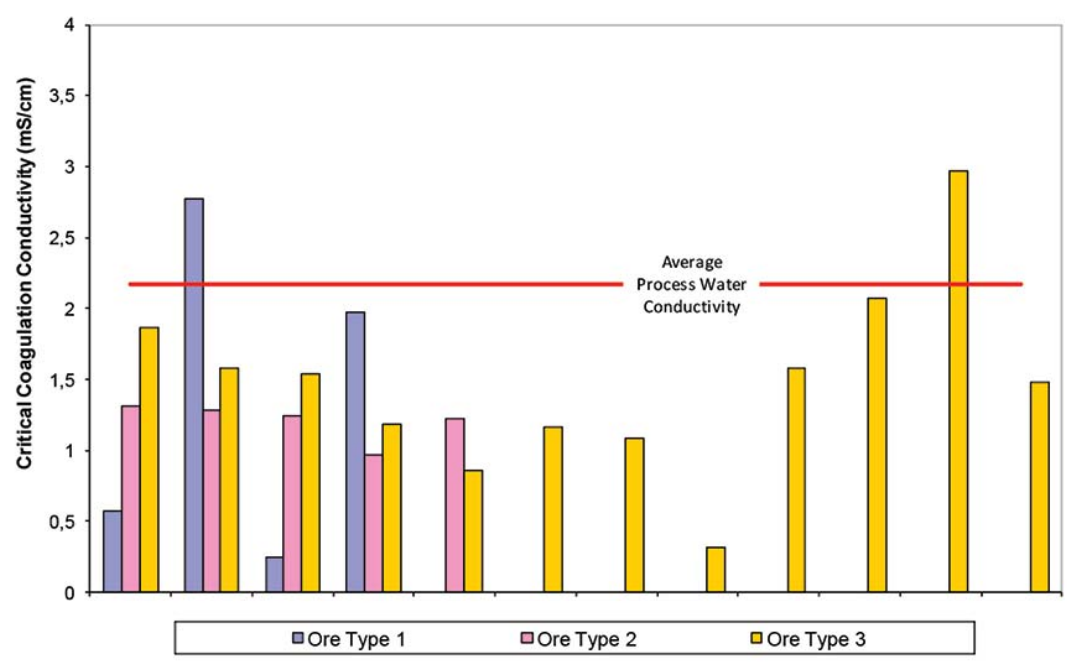

Figure 10-Critical coagulation conductivity requirement and process water conductivity at kimberlite mine $\mathrm{A}$

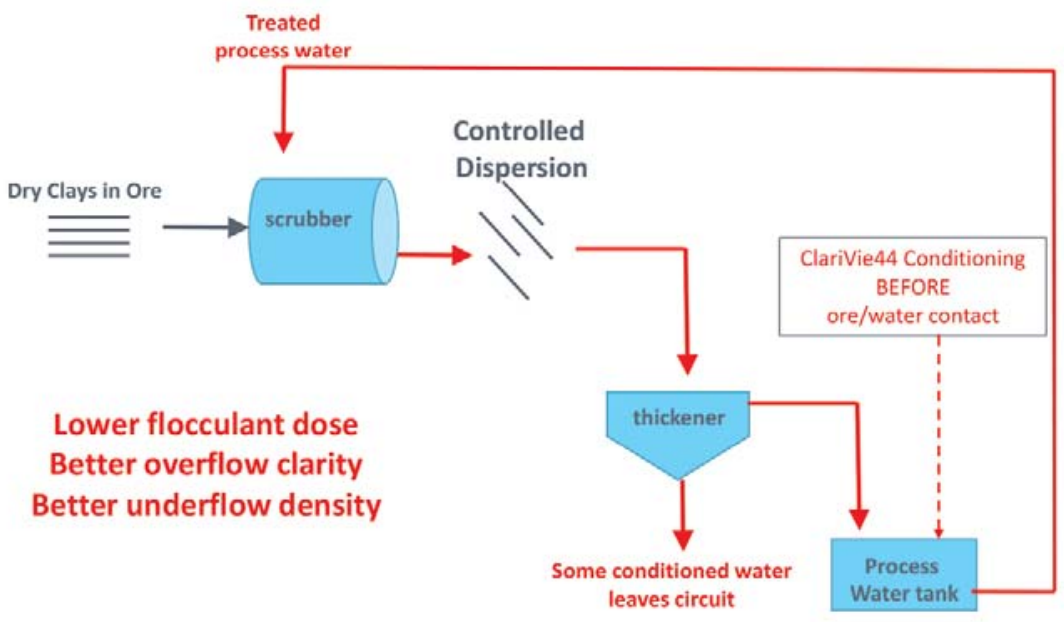

Figure 11-ClariVie44® dosing strategy
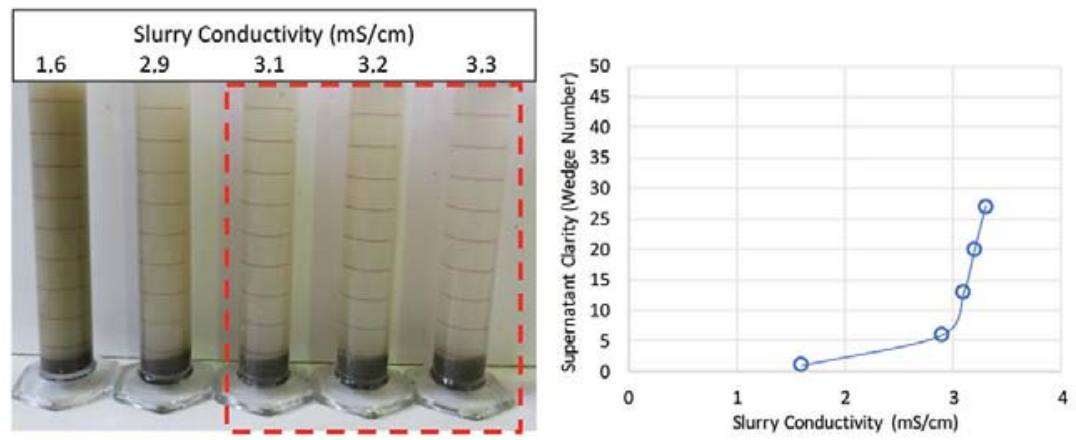

Figure 12-Effect of increasing conductivity on the suspension behaviour of kimberlite mine A slurry (ClariVie44®-treated slurries highlighted)

results showing a clear correlation between increasing process water conductivity and decreasing flocculant consumption (Figure 13).

\section{Discussion}

In general, kimberlite mines can be categorized into two types in terms of tailings slurry colloidal and dispersive properties.
Non-problematic mines-The tailings slurries at these mines are non-dispersive and easily flocculated at relatively low reagent dose. The thickener underflow densities are generally relatively high (depending on the type of dewatering equipment selected), overflow clarities are generally excellent, and water recoveries are good. The metallurgical plant therefore operates 


\section{A strategy for improving water recovery in kimberlitic diamond mines}

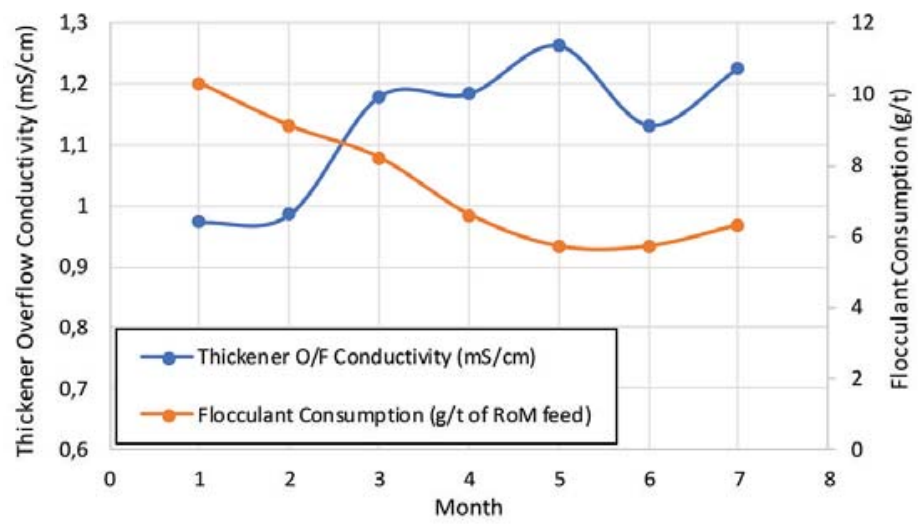

Figure 13-Correlation between process water conductivity and decreasing flocculant consumption at mine B

with clean process water. These mines do not need to further condition the process water as the natural conductivity of the raw water supply is sufficient to prevent uncontrolled clay dispersion. Further improvements in water recovery would only require interventions to optimize reagent make-up and dosing control and/or the thickener discharge control. The latter is not straightforward since the new thickener control philosophy of generating higher underflow densities would need to be compatible with the capabilities of the existing hydraulic tailings discharge infrastructure and the existing TSF operating and management methodology.

Problematic mines-The tailings slurries at these mines are highly dispersive and difficult to flocculate and settle. High flocculant and/or polymer coagulant doses are required before solid/liquid separation will occur, underflow densities are generally low, and water recoveries are generally poor, even after treatment. At these mines the natural conductivity of the raw water supply is too low, and conditioning of the process water would be required to improve water recovery and for returning clarified process water back to the plant. Once the colloidal properties of the slurry have been brought under control, the subsequent strategies for increasing water recovery as described above can be implemented. There are, however, some mines in this category that operate normally by returning unclarified process water to the process plant. This is possible since the viscosity of highly dispersive process water can remain low even up to relatively high solids loadings (10 to $15 \%$ by mass of the $-20 \mu \mathrm{m}$ fraction). Above this solids loading, however, the ultrafine solids begin to influence the water's viscous properties and may affect diamond recovery efficiency in the dense media separation (DMS) circuit. There is a danger, however, that by implementing a process water conditioning strategy at this point, the solids in the process water may in fact 'gel' instead of achieving solid/liquid separation. In this event, the water in the entire process circuit would take on the consistency of yoghurt and would need to be flushed out and replaced with fresh water, thereby dramatically increasing water loss.

\section{Conclusion}

For the kimberlite diamond mine operator, increasing water use efficiency in terms of cubic metres per ton of feed processed means treating more tons with the existing water supply volume or treating existing tons with less water. In both cases, achieving this outcome depends on improving the water recovery efficiency at the dewatering unit process by increasing the density of the tailings being discharged.

The basis of this strategy depends on the colloidal state of the tailings slurry being such that solid/liquid separation takes place naturally. Some kimberlite mines are in the fortunate position of having saline or brackish raw water sources which impart a high conductivity to the plant process water. Under these conditions, the smectite clays in the kimberlitic ore are unable to swell and disperse once in suspension and are flocculated easily at the plant thickener. The mine operator is then able to concentrate on the real drivers for improving thickener underflow density, such as reagent make-up and dosing control and, more importantly, thickener underflow discharge control.

However, at other kimberlite mines where the raw water supply is drawn from low-saline sources, the primary step of solid/liquid separation does not readily occur naturally due to the dispersive slurry properties generated. In these cases, a strategy for increasing the process water conductivity by introducing a water conditioning reagent such as ClariVie44® is required before the subsequent drivers for improving water recovery can be implemented.

\section{References}

GrEGoRY, J. 1989. Fundamentals of flocculation. CRC Critical Reviews in Environmental Control, vol. 19, no. 3. CRC Press. pp. 185-230.

GrimM, R.E. 1968. Clay Mineralogy. 2nd edn. McGraw Hill, New York.

Mering, J. 1946. The hydration of montmorillonite. Transactions of the Faraday Society. vol. 42. pp. 205-219.

Mitchell, J.K. and SogA, K. 2005. Soil-water-chemical interactions. Fundamental of Soil Behaviour. Wiley, Hoboken, NJ. Chapter 6.

RicHARDS, L.A. 1969. Diagnosis and improvement of saline and alkali soils. Handbook no. 60. US Department of Agriculture.

Sequet, H., de la Calle, C., and Pezerat, H. 1975. Swelling and structural organization of saponite. Clays and Clay Minerals, vol. 23. pp. 1-9.

SvARovSKy, L. 1981. Solid-Liquid Separation. 2nd edn. Butterworths, London. Van Olphen, H. 1977. Clay Colloid Chemistry. Wiley, New York. 